\title{
Electronic reminders for intraoperative antibiotic re-dosing
}

\author{
S. Kendall Smith, MD, PhD • Guy de Lisle Dear, MA, MB, BChir, FRCA · Sara H. Cooper, PharmD • \\ Brad M. Taicher, DO, MBA
}

Received: 13 September 2016/Revised: 29 December 2016/Accepted: 17 January 2017/Published online: 27 January 2017

(c) Canadian Anesthesiologists' Society 2017

\section{To the Editor,}

Administration of antibiotics within one hour of surgical incision for qualifying surgeries has become the standard of care for reducing the incidence of surgical-site infections, as described by the United States' Joint Commission Surgical Care Improvement Project. $^{\mathrm{A}}$ Interestingly, although re-dosing antibiotics during prolonged surgical cases is also recommended, the dosing interval is often determined by a hospital's Antimicrobial Stewardship and Evaluation Team (ASET) and may vary by antibiotic and by hospital. ${ }^{1}$ Although anesthesia providers at our institution are aware of these recommendations, and compliance with pre-incisional antibiotic dosing is $>99.9 \%$, a recent convenience sample revealed that our compliance with antibiotic redosing during long cases was sub-optimal [68/88 (77.3\%)]. Furthermore, a preliminary review of the 20 cases that failed to re-dose in a timely manner revealed a multitude of reasons. Most commonly, failure to re-dose could be attributed to (1) concurrent intraoperative activities during the anticipated time of re-dosing and (2) failure to recognize the recommended dosing interval for secondline antibiotics in cases where first-line antibiotics were contraindicated, often because of allergy.

At least two previous studies have demonstrated the utility of real-time intraoperative electronic reminder

S. K. Smith, MD, PhD - G. de Lisle Dear, MA, MB, BChir, FRCA .

B. M. Taicher, DO, MBA ( $\square)$

Department of Anesthesiology, Duke University Medical Center,

Durham, NC, USA

e-mail: Brad.Taicher@duke.edu

S. H. Cooper, PharmD

Department of Pharmacy, Duke University Medical Center,

Durham, NC, USA systems for increasing compliance of timely prophylactic antibiotic re-dosing during prolonged surgical cases. ${ }^{2,3}$ As a quality improvement endeavor, our hospital implemented such a system in our Anesthesia Information Management System (AIMS) (Epic, Verona, WI, USA) with the intent to achieve a similar increase in compliance with timely redosing of intraoperative prophylactic antibiotics. In this era of frequent messages, reminders, alerts, and advisories from our AIMS, however, it was unclear whether implementation of such a system would result in improved compliance. ${ }^{4}$ This preliminary retrospective observational study (deemed exempt for review by our Institutional Review Board, August 2016) aimed to determine whether such a system could improve intraoperative antibiotic re-dosing compliance to $>90 \%$.

A real-time intraoperative electronic reminder system was designed to provide a pop-up message when an antibiotic was recommended to be re-dosed as per intervals set by our ASET guideline. To determine baseline compliance, a convenience sample of all cases in neurosurgical operating suites that required re-dosing of prophylactic antibiotics were examined over a three-month period prior to intervention. In the following nine months, we analyzed the pre-intervention data, formulated a quality improvement plan for automated reminders in our AIMS, presented the plan to an internal Patient Safety and Quality Council, and worked with Epic builders to implement the automated tool. Post-intervention data were collected one month following launch of the tool, which allowed us to mitigate a potential Hawthorne effect. Furthermore, preintervention and post-intervention data were collected during the same academic interval to control for trainee

\footnotetext{
A The Joint Commission. Available from URL; https://www. jointcommission.org/ (accessed December 2016).
} 
Figure A run

chart demonstrates a significant post-intervention process shift.

The blue line represents a postintervention run of compliance rates above the pre-intervention median (green line)

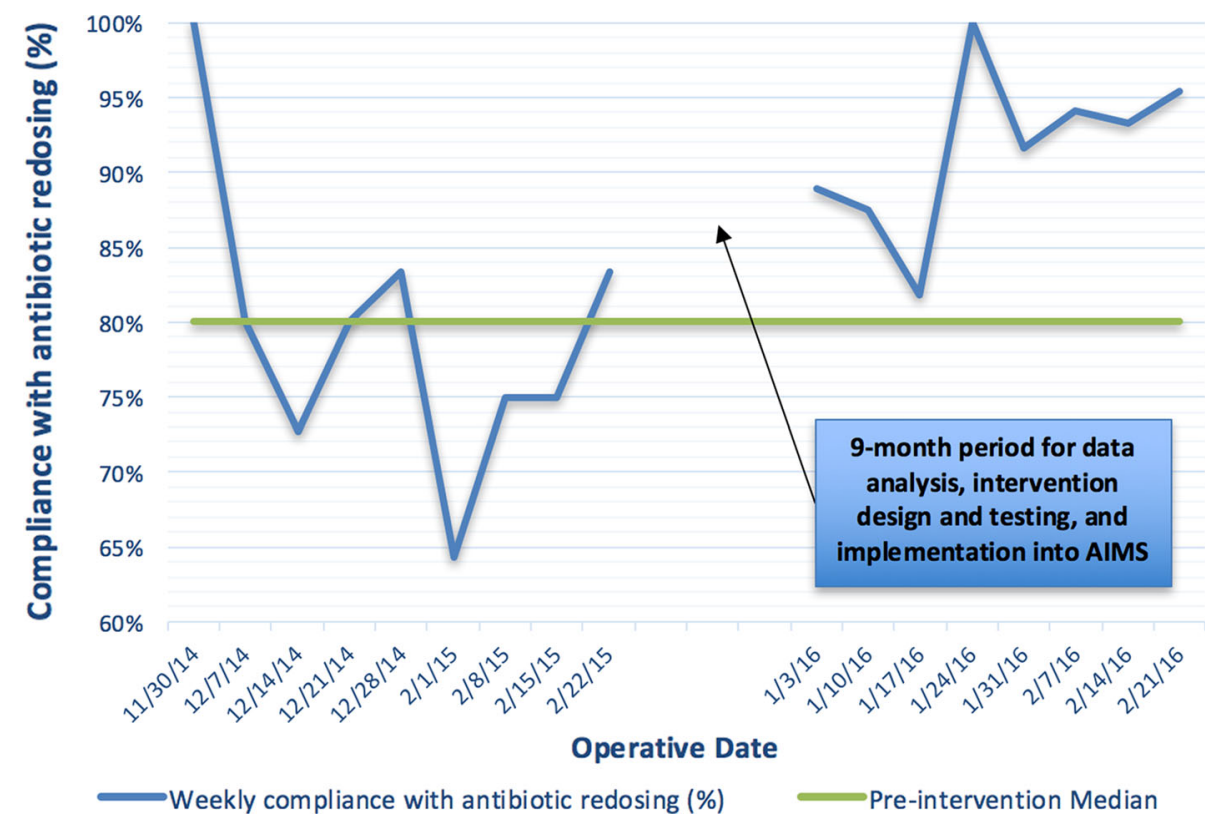

experience levels. We looked at re-dosing compliance for 213 patients who required re-dosing in six neurosurgical operating rooms over the course of 17 weeks ( $n=9$ weeks, pre-intervention; $n=8$ weeks, post-intervention). At our institution, antibiotic re-dosing was defined as compliant if it occurred within $30 \mathrm{~min}$ before or after the recommended time.

Analysis of our preliminary data suggests that implementation of a real-time electronic reminder system in our AIMS was associated with increased mean (standard deviation) compliance of timely re-dosing of antibiotics from $79.3(9.8) \%$ pre-intervention to 91.6 (5.5)\% postintervention [absolute risk reduction, 12.3\%; 95\% confidence interval, 4.1 to $20.5 ; P=0.007$, Student's two-group $t$ test (SAS, Cary, NC, USA)]. A run chart (Figure) demonstrates a significant post-intervention process shift, with the blue line representing a postintervention run of compliance rates above the preintervention median, which is depicted by the green line. ${ }^{5}$

Overall, these results suggest that electronic reminders may help the provider comply with the timely re-dosing of antibiotics during prolonged surgical cases, even in the era of frequent, unrelated electronic messages and advisories. Importantly, future investigations will focus on the systemwide impact, potential association with adverse outcomes including surgical-site infections, and sustainability, which was not directly addressed in this study. Finally, we aim to identify and further characterize the causes of residual noncompliance observed despite implementation of this realtime electronic reminder system.
Conflict of interest None of the authors has any conflict of interest, financial or otherwise, to disclose.

Editorial responsibility This submission was handled by Dr. Steven Backman, Associate Editor, Canadian Journal of Anesthesia.

Funding source None of the authors has any funding sources to disclose.

\section{References}

1. Campbell KA, Stein S, Looze C, Bosco JA. Antibiotic stewardship in orthopaedic surgery: principles and practice. J Am Acad Orthop Surg 2014; 22: 772-81.

2. St Jacques P, Sanders N, Patel N, Talbot TR, Deshpande JK, Higgins $M$. Improving timely surgical antibiotic prophylaxis redosing administration using computerized record prompts. Surg Infect 2005; 6: 215-21.

3. Nair BG, Newman SF, Peterson GN, Schwid HA. Automated electronic reminders to improve redosing of antibiotics during surgical cases: comparison of two approaches. Surg Infect (Larchmt) 2011; 12: 57-63.

4. Rayo MF, Moffatt-Bruce SD. Alarm system management: evidence-based guidance encouraging direct measurement of informativeness to improve alarm response. BMJ Qual Saf 2015; 24: 282-6.

5. Perla RJ, Provost LP, Murray SK. The run chart: a simple analytical tool for learning from variation in healthcare processes. BMJ Qual Saf 2011; 20: 46-51. 